\title{
Multilinguales
}

\section{Quel profil du lecteur-apprenant algérien face à un} mythe?

What profile of the reader-learner algerian face a myth?

$$
\text { ما هو التوصيف الخاص بالقارئ المتعلم الجزائري الذي يواجه أسطورة؟ }
$$

\section{Wassila Soltani}

\section{CpenEdition}

\section{Journals}

Édition électronique

URL : http://journals.openedition.org/multilinguales/1430

DOI : 10.4000 /multilinguales. 1430

ISSN : 2335-1853

\section{Éditeur}

Université Abderrahmane Mira - Bejaia

\section{Référence électronique}

Wassila Soltani, «Quel profil du lecteur-apprenant algérien face à un mythe ?», Multilinguales [En ligne], 9 | 2018, mis en ligne le 01 juin 2018, consulté le 17 septembre 2019. URL : http:// journals.openedition.org/multilinguales/1430 ; DOI : 10.4000/multilinguales.1430

Ce document a été généré automatiquement le 17 septembre 2019.

\section{c) $8 \odot$}

Multilinguales est mise à disposition selon les termes de la Licence Creative Commons Attribution Pas d'Utilisation Commerciale - Pas de Modification 4.0 International 


\section{Quel profil du lecteur-apprenant algérien face à un mythe?}

What profile of the reader-learner algerian face a myth?

$$
\text { ما هو التوصيف الخاص بالقارئ المتعلم الجزائري الذي يواجه أسطورة؟ }
$$

\section{Wassila Soltani}

1 La lecture est, avant tout, une rencontre entre un sujet et un texte qui aboutit à une construction d'un sens dans un contexte particulier. C'est un moment opportun pour l'apprentissage. D'ailleurs, le déploiement des compétences lectorales n'est pas un préalable à la lecture mais une suite de celle-ci. C'est par la lecture de textes divers que les apprenants acquièrent les instruments leur permettant de s'approprier les textes, d'en appréhender le sens, de s'entrainer aussi à distinguer la rationalité de l'émotion et à identifier les valeurs qui structurent un discours et forment leur esprit critique.

2 Bien souvent, la lecture se présente comme un défi pour le lecteur-apprenant qui se trouve sans cesse sollicité et invité à rencontrer des objets de savoirs complexes nécessaires à la construction de ses apprentissages. Afin d'examiner plus minutieusement certains aspects de la lecture-réception des textes littéraires (notamment le mythe en tant que forme culturelle), le présent article propose une brève étude expérimentale menée au sein d'une université algérienne et met l'accent sur les principales contraintes liées au fait lectoral et à la posture intersubjective des lecteurs face à des altérités.

\section{La lecture littéraire ou la (re)construction du sens}

Centrale est la question de la lecture au sein d'une classe de FLE. Pour longtemps, les méthodes de son enseignement balançaient entre choix raisonné ou aléatoire dans l'espoir de mener à bien cette activité (DAUNAY, 2007). Il est vrai que pour la lecture littéraire, les défis sont plus grands vu la complexité des textes « d'art ». En fait, c'est le caractère dit « littéraire » qui rend la lecture des œuvres encore plus problématique vu que ce qualificatif implique souvent des compétences transversales. 
4 Lire un récit fictionnel «engage le lecteur à adopter une attitude de coopération " (ECO, cité par DUMORTIER, 2001: 126). Appelé à compléter les lacunes ou les «lieux d'indétermination » (ISER, $1985: 48$ ) du texte, le lecteur réanime ses acquis antérieurs afin de saisir une éventuelle portée symbolique du texte. Comment définir, alors, la lecture littéraire? Cette question, redoutable dans sa simplicité, appelle à des réponses différentes en fonction du texte lui-même qui peut être abordé comme "une œuvre d'art, une communication linguistique ou une structure qui ne prend sens que dans la réception." (TOURSEL et VASSEVIERE, $2010: 21$ ). Selon PICARD, la lecture littéraire se reconnait à trois fonctions :

La première est «la subversion dans la conformité ». Le texte littéraire à la fois conteste et suppose une culture. [...] La seconde fonction est «l'élection du sens dans la polysémie». Le texte littéraire renvoie toujours à une pluralité de significations. [...] La dernière fonction [...] est « la modélisation par une expérience de réalité fictive ». Il est ici question du rôle pédagogique de la lecture. (PICARD, cité par JOUVE, 1993 : 104).

5 Ce qui caractérise la lecture littéraire, c'est qu'elle n'a pas un rôle fonctionnel : sa priorité n'est pas l'information mais plutôt l'examen critique et appréciatif. C'est pour cela que la littérature implique une réception particulière. Autrement dit, l'espace littéraire suscite des réactions d'adhésion ou de rejet de l'objet lu. Cette opération traduit des tensions entre lecture impliquée (investie) et lecture distanciée et renvoie, en quelque sorte, à des postures possibles qu'un lecteur peut manifester face à un texte.

D'un point de vue pédagogique, il faut, aussi, souligner l'importance d'une

sensibilisation aux conventions génériques (CANVAT, 1999) et à la distance culturelle (COLLES, 1994). Par défaut de sensibilité aux caractéristiques du récit relevant de ces conventions ou s'expliquant par cette distance, le lecteur adolescent interprétera l'univers de l'histoire en se référant exclusivement à ses valeurs et à ses normes de comportement. (DUMORTIER, $2001: 156$ ).

7 L'apprenant lit le texte avec ses propres valeurs et s'investit sur le plan affectif. C'est pourquoi, toute planification pédagogique doit prendre en considération le rôle primordial du contexte dans l'interprétation des œuvres littéraires.

8 Le texte littéraire est un objet polymorphe, ouvert et inachevé. En ce sens, que peut apporter la lecture littéraire en matière d'apprentissage des langues étrangères ? La réponse est évidente, beaucoup, pour ne pas dire tout le savoir nécessaire pour se former sur les deux plans linguistique et culturel. En lisant, l'apprenant se positionne en tant que sujet indépendant, différent d'un Autrui véhiculé par le fond culturel du texte. En ce sens, il s'agit de s'ouvrir sur l'Altérité et d'accéder à la culture universelle : "Le texte littéraire, en tant qu'outil de médiation de connaissance entre le monde et le lecteur, participerait à la formation du sujet, à la fois par les regards qu'il porte sur l'autre et sur luimême dans l'acte de lire. ». (LEBRUN, 2005).

9 La lecture littéraire permettrait à l'apprenant de s'épanouir tant sur le plan intellectuel qu'affectif car » la connaissance de la littérature n'est pas une fin en soi, mais une des voies royales conduisant à l'accomplissement de chacun » (TODOROv, 2007: 25). Elle intervient, aussi, dans la construction de l'identité narrative (RICOEUR, 1985) du lecteur :

Lire, c'est devenir [...]. La lecture d'un texte implique donc toujours, à un plus ou moins grand degré, une opération qu'on ne peut pas appeler autrement qu'ontologique. Pour le temps où elle s'accomplit, elle entraîne une transformation si radicale de la pensée lectrice. (POULET, 1975 : 66-67). 
10 Il semble que l'intérêt pédagogique du texte littéraire est confirmé. Cependant, il semble que son usage effectif ne soit pas aussi simple qu'on le croit. Le hiatus entre les réflexions théoriques et les pratiques de terrain est de vigueur. Le lecteur n'a pas encore pu décrocher son statut mérité et sa subjectivité demeure discutable. Les lectures plurielles (cf. MORA et SEGUY, 1978) apparaissent peu dans les dispositifs didactiques et les nouvelles approches relatives à la lecture littéraire dans sa dimension trans(inter)culturelle sont encore moins appliquées ou peu maîtrisées.

\section{La problématique de l'implicite}

La littérature est, en effet, « un discours assorti d'un silence, un discours qui désigne un espace muet, un espace qui n'est pas les mots, qui est d'un autre registre que celui du langage » (GRIMAUD et RIOU, $2012:$ 74). Parler de «l'espace sacré » du texte, c'est mettre l'accent sur les textes résistants. D'ailleurs, «un texte qui résiste n'est pas nécessairement un texte compliqué, mais c'est un texte complexe, c'est celui qu'on n'en finit pas de comprendre, qui n'a jamais tout dit et qui continue de résonner. » (Ibidem). En d'autres termes, derrière certains dits se cachent encore d'autres paroles non dites. Ainsi, le texte littéraire s'apparente à des lectures plurielles, du coup, à plusieurs significations possibles. Néanmoins, nul n'a à prétendre que tel sens est le «bon » ou le «meilleur ». Voilà ce qui fait du texte littéraire un objet hautement complexe.

Bien avant l'éclatement des genres qui caractérise les écrits contemporains, la visibilité du texte littéraire a été garantie par l'identification du genre et la soumission à certaines règles déterminant chacune des catégories narratives. Mais que faire, maintenant que le texte moderne transgresse certaines normes de la lisibilité textuelle, qu'il brouille les frontières ? Qu'en est-il de la littérarité, caractéristique fondamentale du texte littéraire, à l'aire où le parler ordinaire, l'oralité et le patrimoine local traversent, sans cesse, les productions littéraires contemporaines?

13 Ces questionnements ont accentué le dilemme du choix du support pour une lecture littéraire analytique qui devient de plus en plus un moment décisif. Travailler un texte réticent en classe, c'est s'apprêter à confronter une réalité délicate. Celle de l'épreuve relative à l'implicite étant donné que "dans chaque énoncé, oral ou écrit, il y a une grande part d'implicite, il y a "plus de vide que de plein", plus d'informations tues que d'informations dites.» (ROCQUET, $2002: 1$ ). Et c'est cet espace muet qui «retient le lecteur par l'émotion » (GRIMAUD et RIOU, op.cit. : 78) et fait l'objet de la quête interprétative. A la manière du bogolan ${ }^{1}$, le lecteur doit combler des blancs du texte afin d'en trouver le sens. Sur ces entrefaites, comment apprendre aux élèves à comprendre l'implicite des œuvres littéraires? Si, pour certains, l'interprétation commence là où s'arrête la compréhension (MASSEAU, 2017), peut-on supposer que chaque lecteur qui a bien compris un texte pourrait l'interpréter?

Souvent la compréhension est associée au sens premier, littéral, dénoté ou explicite, tandis que l'interprétation renvoie au second sens, connoté ou implicite. Il en ressort que l'interprétation est une activité seconde à la compréhension mais plus élitiste et supérieure à elle. Dans une perspective proche, BARTHES précise qu'« interpréter un texte, ce n'est pas lui donner un sens (plus ou moins fondé, plus ou moins libre), c'est au contraire apprécier de quel pluriel il est fait.»(BARTHES, 1970: 11). En d'autres mots, si la 
compréhension d'un texte sert à élucider ses énigmes et à lui attribuer un sens, l'interprétation participe à le faire revivre.

FALARDEAU, de son côté, avance une thèse, que nous approuvons également, quant à la complémentarité des deux opérations qui

agissent en concomitance, l'une puisant dans les signes de 1'autre. Ce sera là la définition d'une lecture littéraire riche, productive: les informations comprises sont appelées à être interprétées, les deux registres devant ainsi être présentés dans leur concomitance dès le premier apprentissage de la lecture. (FALARDEAU, $2003: 187)$.

16 Dans un autre ordre d'idées, DUfAYs conçoit le processus de compréhension sous un angle distinct. Pour lui : " comprendre un texte [...] c'est avant tout y reconnaitre des stéréotypes, des structures de sens abstraites et durablement inscrites dans la mémoire collective. Cette fonction cognitive de première importance se double d'une fonction axiologique. " (DUFAYS, 1994 : 350). L'évaluation d'un texte est déterminée par l'attitude $\mathrm{du}$ lecteur face aux modalisations référentielles que renferment les séquences structurelles du texte.

Dans le cadre de la classe, l'étude de l'implicite peut introduire des enjeux de socialisation en débattant, par exemple, des lectures plurielles d'un texte donné. Cependant, l'expérience de la lecture, celle impliquée, peut conduire le lecteur à s'aventurer dans un univers ouvert à l'infini. C'est pourquoi l'enseignant doit veiller à ce que le flot des interprétations possibles ne soit synonyme d'une juxtaposition des subjectivités en marge du texte et à ce que les apprenants ne transgressent « les limites de l'interprétation » (ECO, $1992: 17)$.

\section{Effet et réception des personnages fictifs}

La notion de "personnage » est une donnée inhérente à la lecture littéraire. Le mot personnage recouvre plusieurs variétés : il peut renvoyer à des idées ou simplement à des éléments de décor, c'est pourquoi Jouve définit le personnage comme "un tissu de mots » (JOUVE, 1998 : 171).

Dans son ouvrage dédié à l'étude exclusive de cette notion "L'Effet-Personnage », JOUVE distingue trois « régimes de lecture » correspondant aux différents modes de réception du personnage fictif :

"le lectant» renvoie au lecteur qui ne se laisse pas tromper par "l'illusion romanesque » et préfère adopter une attitude réflexive en abordant un texte. Le personnage est reçu comme une instance narrative ou intellectuelle ;

«le lisant» concerne la part du lecteur trompé par le mirage narratif, donc, le personnage est reçu comme une personne. Cet effet est la source du plaisir de lire et « indispensable à la construction du moi »;

«le lu» correspond à «la relation du sujet à lui-même, du moi à ses propres fantasmes ", c'est-à-dire, l'effet dégagé de la lecture peut libérer des tensions inconscientes.

20 Force est de constater que l'impact de ces « êtres de papier $»^{2}$ dépasse l'univers textuel. Certes, nous n'allons pas dresser un historique du développement de cette notion et l'apport des théories critiques en la matière, mais plutôt, nous allons focaliser notre attention sur la part de «l'ère du soupçon » (1956), pour reprendre le titre de Nathalie 
SARRAUTE, dans la métamorphose du personnage littéraire. Le nouveau roman opère une déconstruction maximale de l'identité référentielle des personnages. Des êtres dotés d'un nom, d'une passion et des portraits détaillés, moteur de l'action narrative, aux personnages ambigus, en crise et sans repères. Bref à des entités perceptives d'un univers problématique, qui traduisent justement l'ère moderne. C'est là encore un autre aspect ${ }^{3}$ qui complique davantage la maîtrise de la lecture littéraire.

21 A la question de la construction des personnages succède celle de leur réception. Cette dernière devient difficile à cause des personnages difficiles à identifier par leur caractère énigmatique (absurdité inédite, incertitude, vide émotionnel...). Cependant, ce type de personnage apporte, à vrai dire, une remise en question et un retour sur les débats autour de la condition humaine. Sur le plan des apprentissages, ces actantsproblématiques suscitent l'intérêt des lecteurs et servent, des fois, à une catharsis : «Les personnages me font accéder à mon tour au grand règne des métamorphoses. [...]. En lisant, je me livre, je m'oublie; je me compare; je m'absorbe, je m'absous. Sur le modèle et à l'image du personnage, je deviens autre.» (SALLENAVE, 1991 : 132-134). Sur ces entrefaites, le rôle du personnage demeure crucial dans l'acte de lire comme le souligne TAUVERON : «Le personnage est un axe essentiel de la lecture du récit; un facteur de rappel et de progression qui offre au lecteur la possibilité de construire son interprétation et revêt différentes fonctions (mimétique, symbolique, pragmatique etc.) » $(1995: 14)$.

D'ailleurs, la lecture et la réception du geste des personnages littéraires peuvent être conditionnées par deux critères essentiels: d'une part, l'expérience personnelle du lecteur et d'autre part, les représentations communes déterminées par le contexte de la lecture. Mais cette dernière est, aussi, influencée par la manière dont un personnage est peint dans l'œuvre ${ }^{4}$. Il apparaît que la réception n'est pas relative à des compétences, comme on a tendance à le croire, mais plutôt à des besoins, des choix, et à des attitudes individuelles.

Il y a, donc, tout un intérêt d'une réflexion sur la fonction du personnage en tant qu'outil pédagogique et un élément qui motive l'apprenant pour aborder le texte littéraire. De même, le personnage peut être appréhendé tel un support de l'axiologisation ${ }^{5}$.

\section{Lire des textes sous l'angle du mythe}

Dans une tentative de répondre à nos préoccupations, nous avons élaboré un dispositif didactique dont les termes clés sont: cercles de lecture interactive, approche comparative et interprétative des mythes. C'est au sein d'un atelier de lecture que nous avons proposé une lecture de deux romans contemporains (Qui se souvient de la mer de Mohammed DIB et N'Zid de Malika MOКкEDEM) véhiculant une réécriture moderne d'un ou de plusieurs mythes.

L'atelier de lecture a eu lieu à l'Université de Mohamed Khider à Biskra, au sud algérien, avec une promotion de Master I Littérature. Il s'est étalé sur tout un semestre dans le cadre d'un module intitulé "Mythes, cultures et sociétés». Le groupe expérimental se compose de trente deux étudiants (dix jeunes hommes et vingt deux jeunes femmes). Plus d'une quinzaine de ces étudiants habitent le centre ville et le reste viennent de la périphérie (Des régions environnantes de la wilaya). Pour ce qui est de 
leur statut social : une grande majorité de ces étudiants sont déjà fonctionnaires (des enseignant(e)s, une infirmière, un inspecteur de l'éducation au primaire (nanti d'une licence classique et non d'une licence LMD), deux retraités, un bijoutier). Le reste, ce sont des jeunes ayant entre 22 et 28 ans. Le milieu social des étudiants varie, surtout suivant la résidence, balançant entre favorisé et moins favorisé pour ne pas dire défavorisé. Etant tous de confession musulmane, ces étudiants partagent les mêmes croyances, pratiquées différemment (ceux issus de la périphérie sont plus réservés et attachés à leurs croyances religieuses si l'on tient compte de leurs propos et réactions durant les débats). De même pour leur « répertoire culturel », les étudiants partagent presque le même imaginaire culturel, imprégné des mêmes contes et légendes, soumis aux mêmes traditions.

En fait, selon notre protocole expérimental, il demeure important de prendre en considération les caractéristiques hétérogènes ${ }^{6} \mathrm{de}$ nos enquêtés pour expliquer les nuances dans la réception des mythes grecs ainsi que les représentations socioculturelles qui émanent de cette dernière.

Selon l'esprit de cette démarche, les apprenants doivent, essentiellement, faire appel à la mythocritique ${ }^{7}$. Cette approche vise à mettre en évidence, dans une œuvre littéraire, les mythes directeurs et leurs transformations significatives. Ces "mythes littéraires", qu'un écrivain peut modifier à sa guise, forment une matrice génératrice de sens et facilitent l'interprétation du texte. Ce projet se base sur un postulat simple : si le fond référentiel du mythe est souvent rejeté (dans un contexte arabo-musulman) à cause d'un filtrage religieux ou axiologique, la transposition du mythe dans un contexte moderne qui simule, plus ou moins, la vie réelle des apprenants pourrait atténuer les problèmes liés à sa réception. Ainsi, les pré-acquis des apprenants, même subjectifs, pourraient se transformer en un ancrage objectif en multipliant les expériences de lecture. Dans ce sens, il s'agit d'étudier le personnage mythique en tant que médiation symbolique et culturelle et non comme une construction narrative.

La finalité de la lecture interactive est doublement articulée : faire dialoguer autour des œuvres. En d'autres mots, faire intervenir des textes antérieurs (par processus d'intertextualité) puis les lier dans des réseaux de signification avec le texte en question ce qui déclenche une confrontation des hypothèses interprétatives. Pour ce faire, se référer à son propre répertoire culturel pourrait mener le lecteur à mieux comprendre et interpréter un mythe en établissant des ponts entre sa culture d'origine et celle ciblée. D'ailleurs, ce lecteur n'est pas «Modèle» (ECO, 1985) mais un lecteur ordinaire, un sujet qui a sa manière de lire et sa subjectivité propre. Et c'est justement, le débat autour des textes lus qui devient, d'abord "un lieu d'intersubjectivité» (TAUVERON, $2002: 21$ ), puis, un espace possible d'objectivation des interprétations jugées «trop subjectives » ou déroutées par des stéréotypes d'ordre culturel, esthétique, ou moral.

Comme point de départ, et dans le but d'exploiter les points d'incertitude des textes choisis, nous avons demandé aux participants de lire, chacun, un extrait qu'il juge opaque, puis la communauté interprétative accède à une réflexion collective autour de l'extrait. La grille suivante présente un exemple : 
Amina : Pour ma part, en lisant cet extrait [elle lit le passage suivant] :» Avec ses lumières pompières et ses murmures trompeurs, la mer infantilise les marins. Les promesses de ses bleus les aspirent. Ses mirages les inspirent. Ils s'y jettent comme s'ils se précipitaient dans le vide avec parfois l'envie de se perdre, de tout perdre. Mais la mémoire les tient aux pattes et aux nerfs comme des marionnettes. Elle peut les rembobiner quand elle veut pour les recracher dans leurs désillusions. Au moment même où ils croient braver, en héros, et la mémoire et la mer, ils ne sont guère que des zombies qui parlent aux baleines, aux méduses et aux oiseaux égarés. Des gueux, sans terre. » N'Zid (188-189), j'ai dû m'arrêter, c'est confus !

Youcef : Ça parle de l'errance non! trop de jeux de mots, compliqué ouff !

Sara : Mais, non! c'est une réécriture d'un épisode du mythe d'Ulysse.

Youcef : Comment ça?

Sara : Regardez ces mots : murmures trompeuses (c'est le champ des sirènes), la mémoire les tient aux pattes (Ulysse attaché au navire).

Amina : Mais, Mokeddem substitue les cordes à la mémoire, c'est elle qui empêche les marins de se laisser séduire par le désir éphémère.

Rahma : Qui dit mémoire, dit origines et passé. Donc tout comme Ulysse est assoiffé de rejoindre sa patrie, ce passage évoque la situation des exilés qui se trouvent entre deux espaces contradictoires : rester ou partir!

Amina : A la fin de l'extrait cette phrase » des gueux sans terre » cela veut dire que Nora s'est perdue en plein mer, elle a perdu son identité ?

Sara : Le contraire, je vois que le fait de choisir la Méditerranée (lieu ouvert, sans frontières) c'est justement une aspiration à un monde tolérant où des identités multiculturelles peuvent se côtoyer et s'enrichir.

Youcef : Apparemment, cet extrait dit plus que je l'ai cru!

Il n'est guère question de nier la subjectivité des lecteurs mais le débat vise à pousser l'apprenant à accéder à l'altérité des œuvres lues, à éviter les jugements extrémistes et les réactions abusées qui accompagnent l'expérience lectorale. Il s'agit de passer de l'expression du Moi à une socialisation du texte.

31 Par la suite, deux activités ont été proposées aux apprenants: une, portant sur l'identification des mythes réécrits dans les séquences narratives des deux romans, l'autre se présente sous forme d'un questionnaire de compréhension. La grille cidessous est représentative de la $1^{\text {ère }}$ activité de l'atelier de lecture. Les participants ont été appelés à lire des extraits choisis des deux romans, puis identifier les mythèmes qui renvoient aux mythes réécrits. Les pourcentages indiquent la totalité des variables « oui =succès », « non =échec » repérés dans l'ensemble des copies des apprenants.

Tableau $\mathrm{n}^{\circ} 1$ : Tableau récapitulatif de la première activité

Premier roman : «Qui se souvient de la mer » Mohammed Dib

Extraits

\begin{tabular}{|l|l|l|l|}
\multicolumn{2}{|l|}{$\begin{array}{l}\text { Identification des } \\
\text { mythèmes }\end{array}$} & \multicolumn{2}{|l|}{$\begin{array}{l}\text { Identification } \\
\text { des mythes }\end{array}$} \\
\hline Oui & Non & Oui & Non \\
\hline
\end{tabular}




\begin{tabular}{|c|c|c|c|c|}
\hline $\begin{array}{l}\text { "Les épouses, les enfants allèrent quémander la vérité sur le sort } \\
\text { des leurs à toutes les portes. [...] ils pleurèrent[...],supplièrent, et } \\
\text { les femmes tombèrent à genoux devant les minotaures placés en } \\
\text { sentinelles. Leur baisèrent la main. [...]Un air de flûte donnait des } \\
\text { cornes contre les jambes, contre les ventres, frêle mais têtu. Les } \\
\text { minotaures les repoussèrent sans comprendre un traître mot à } \\
\text { leur baragouin. "QSM (p.16) }\end{array}$ & $66,66 \%$ & $33,33 \%$ & $\begin{array}{l}91,66 \\
\%\end{array}$ & $8,33 \%$ \\
\hline $\begin{array}{l}\text { "En effet, sommes-nous plus qu'un dédale à l'intérieur d'un autre } \\
\text { dédale à présent, abstraction faite de la faculté que nous avons de } \\
\text { nous déplacer? Certes, le plus reculé de ses couloirs les plus } \\
\text { tortueux possède, à coup sûr, plus d'animation, plus d'idées, que le } \\
\text { cerveau le plus actif du plus ingénieux de ses habitants. Chacun, } \\
\text { chaque galerie s'entend, sa vie propre, reliée toutefois par } \\
\text { d'invisibles fils au reste, notamment au centre nerveux que nous } \\
\text { nommons Médresse » QSM (p.22) }\end{array}$ & $\begin{array}{c}37,5 \\
\%\end{array}$ & $62,5 \%$ & $\begin{array}{l}50 \\
\%\end{array}$ & $\begin{array}{c}50 \\
\%\end{array}$ \\
\hline \multicolumn{5}{|l|}{ Deuxième roman : « N’Zid » de Malika Mokkedem } \\
\hline $\begin{array}{l}\text { "Je ne voudrais pas vous accabler. Mais quand même. Vous portez } \\
\text { la tragédie dans l'œeil et jusque dans la racine des cheveux[...]. } \\
\text { Maintenant on peut rencontrer en pleine mer des Ulysse tout en } \\
\text { crinière, en croupe, en poitrail et le noir fiché dans l'œil et au front. } \\
\text { C'est foutu!» N'Zid (p. 77) }\end{array}$ & $\begin{array}{l}75 \\
\%\end{array}$ & $\begin{array}{l}25 \\
\%\end{array}$ & $79.16 \%$ & $20.83 \%$ \\
\hline $\begin{array}{l}\text { "Je peux connaître votre nom? Elle observe son air pince-sans-rire } \\
\text { et sourit :-Ghoula. Un surnom d'enfance. En arabe, ça signifie " } \\
\text { ogresse ". "N'Zid (p.49). " "Hagitec-magitec" Zana, tu prononces } \\
\text { toujours cette formule avant tes contes d'Algérie. Des histoires de } \\
\text { Djaha, de [...] Ghoula. Moi, je dis "Hagie-magie“.Ça te fait rire" } \\
\text { Hagitec-magitec", maintenant, je me souviens de leur } \\
\text { signification: Je te conte sans te venir?» N'Zid (pp. 102-103) }\end{array}$ & $\begin{array}{c}87.5 \\
\%\end{array}$ & $\begin{array}{c}12.5 \\
\%\end{array}$ & $\begin{array}{l}91.66 \\
\%\end{array}$ & $\begin{array}{l}8.33 \\
\%\end{array}$ \\
\hline
\end{tabular}

De cette première activité, il est manifeste que le repérage des sélections contextuelles permet d'interpréter les textes en fonction de l'unité sémantique identifiée à travers l'isotopie ${ }^{8}$. Nous pensons, les isotopies, ici, comme, renvoyant aux mythèmes qui facilitent l'identification du mythe et renforcent les hypothèses interprétatives. Il s'agit, en quelque sorte, de repérer les "déictiques sémantiques " qui constituent un espace de coréférence culturelle. Certains lecteurs se donnent à imaginer «les scénarios communs et intertextuels» (JouvE, 1993 : 59) entre les différents extraits afin de repérer le mythe en question. Mais «l'hypercodage rhétorique et stylistique» (Ibid. : 58) de certains textes rend, des fois, la compréhension problématique.

La deuxième activité vise, quant à elle, à démontrer à quel point la lecture impliquée/ distanciée peut faciliter ou compliquer le processus d'interprétation en examinant le rôle de la recontextualisation des mythes dans le dépassement des préjugés et le développement de l'esprit critique. Nous nous contentons de présenter ces trois exemples :

A quoi le fil d'Ariane peut être comparé dans «Qui se souvient de la mer »? 
A/Le premier est d'ordre thématique : citons cet exemple Saâdia : « Le thème universel (la guerre), le crime, la monstruosité, le Minotaure, les femmes qui pleurent avec leurs bébés. »

$\mathrm{B} /$ Le deuxième est d'ordre esthétique : donnons cet exemple Rym : « Les deux offrent une représentation poétique du Minotaure, ils imitent la réalité spatiale mais d'une manière inédite, l'un par son écriture de génie qui transgresse la forme d'écriture traditionnelle, et l'autre par son art mimique et son cubisme. ».

D’Ulysse à Sindbad le marin, quelles divergences/convergences possibles, pouvez-vous en déduire?

Les réponses des apprenants ont montré que l'errance, les voyages marins, les aventures fantastiques, la rencontre avec des monstres dont le cyclope sont des éléments communs entre l'histoire d'Ulysse et celle de Sindbad. Quant aux points de divergences repérés dans les copies des participants à l'atelier, nous les présentons sous forme d'une grille :

Tableau $n^{\circ} 2$ : Grille comparative de deux personnages fictifs

\begin{tabular}{|c|c|c|}
\hline & Ulysse & Sindbad le marin \\
\hline Le destin & $\begin{array}{l}\text { Condamné par Poséidon, dieu de la mer, à } \\
\text { errer }\end{array}$ & Il est parti de son propre gré \\
\hline L'âge & adulte & jeune \\
\hline Le statut social & Guerrier et roi & Pauvre bonhomme \\
\hline L'appartenance & Un grec & Un oriental (persan) \\
\hline Le lieu d'aventures & L'océan indien & L'Afrique, le Sud d'Asie \\
\hline $\begin{array}{l}\text { La source de } \\
\text { l'histoire }\end{array}$ & L'Odyssée d'Homère & $\begin{array}{l}\text { Les Mille et une Nuit } \\
\text { (anonyme) }\end{array}$ \\
\hline $\begin{array}{l}\text { L'animal de } \\
\text { compagnie }\end{array}$ & Un chien fidèle & Un corbeau intelligent! \\
\hline
\end{tabular}


Ces résultats permettent de révéler que la prédominance des aspects psychoaffectifs et des références à des modèles relevant du répertoire socioculturel des lecteurs constitue un marquage important. Pour cela, on ne peut ignorer la question des valeurs et de l'investissement émotionnel des apprenants dans la didactique de la lecture littéraire car c'est dans la relation qu'il entretient avec l'Autre qu'un sujet affirme son système de valeurs. En ce sens, en comparant les personnages mythiques à des figures relevant de l'imaginaire collectif des lecteurs (Méduse/Ghoula), (Ulysse/Sindbad), (Cyclope/ Antéchrist), la lecture et la réception de ces mythes semblent être mieux réussies. besoins d'apprentissage langagier et socioculturel, surtout en contact des altérités. En nous basant sur cette étude, nous pouvons systématiser le profil du lecteur algérien comme suit :

il a déjà développé, en contexte scolaire et parascolaire, des représentations et des stéréotypes qui fonctionnent comme des médiateurs symboliques ;

ses procédures de lecture diffèrent selon la thématique et le contenu du texte le situant ainsi entre objectivité et subjectivité. Néanmoins, le subjectivisme prend toujours le dessus quand il est question de réalités d'ordre axiologique ou religieux;

le recours à la langue-culture maternelle est une stratégie récurrente dans l'identification des contenus opaques et plurivoques. à la lisière de l'imaginaire et du symbolique, appelle à une lecture impliquée car les valeurs socioculturelles de l'apprenant déterminent largement l'interprétation de la matière mythique. Entendue comme un espace de transitions, la lecture du mythe permet d'interroger inlassablement le sens. Du coup, proposer une modélisation opérationnelle (recontextualisation) du mythe en l'appliquant aux catégories de l'enseignement-apprentissage des langues étrangères est indispensable. Pour ce faire, les praticiens se doivent de repenser leurs démarches par l'adoption, par exemple, d'une lecture participative ou affectivo-identitaire faisant appel à des compétences qui sollicitent la motivation et l'implication émotionnelle des apprenants. 


\section{BIBLIOGRAPHIE}

BARTES, Roland, S/Z, Seuil, Paris, 1970.

DAUNAY, Bertrand, » État des recherches en didactique de la littérature ", in Recherche en Education, Revue française de pédagogie, № 159, Avril-Juin 2007.

DUFAYS, Jean-Louis, Stéréotypes et lecture, coll. » Philosophie et langage », Liège, Mardaga, 1994.

DUMORTIER, Jean-Louis, Lire le récit de fiction, Pour étayer un apprentissage : théorie et pratique, De book Duculot, Bruxelles, 2001.

ECO, Umberto, Les limites de l'interprétation, Grasset, France, 1992.

FALARDEAU, Erick, «Compréhension et interprétation : deux composantes complémentaires de la lecture littéraire ", in revue des sciences de l'éducation, Volume 29, numéro 3, 2003, pp. 673-694. FOURTANIER, Marie- José, « L'apprentissage du FLE par un support multimodal : l'album comme « texte de lecteur » », in Synergies Brésil n 10, 2012, pp. 23-34.

GRIMAUD, Lorine et RIOU, Chantal, "L'espace sacré du texte ", in Mythes et éducation, sous la direction de LERBET-SERENI, Frédérique / VIALLE, Franck, Savoir et Formation, L'Harmattan, Paris, 2012, pp. 71-85.

HÉNAULT, Anne, Les enjeux de la sémiotique, PUF, 1993.

ISER, Wolfgang, L'acte de lecture, Mardaga, Bruxelles, 1985.

JOUVE, Vincent, L'Effet-Personnage dans le roman, PUF, Paris, 1998.

JOUVE, Vincent, La lecture, Hachette, Paris, 1993.

LE MANCHEC, Claude, L'Adolescent et le récit : Pour une approche concrète de la littérature de jeunesse, L'Ecole, Paris, 2000.

LEBRUN, Marcel, Des technologies pour enseigner et apprendre, 3ème édition revue et augmentée, De Boock, Bruxelles, 2005.

MASSEAU, Yannick, « L'interprétation. Trois questions sur l'interprétation », in Philopsis : Revue numérique, http://www.philopsis.fr

MORA Gilles et SEGUY A., « Lectures plurielles - Approches théoriques et implications pédagogiques ", in Repères pour la rénovation de l'enseignement du français à l'école élémentaire, $\mathrm{n}^{\circ} 48$, 1978.

POULET, Georges, « lecture et interprétation du texte littéraire », in Qu'est-ce qu'un texte ? Ouvrage collectif, Corti, Paris, 1975.

RIALLAND, Ivanne, « La mythocritique en questions », in Questions de Mythocritique, Dictionnaire, sous la direction de CHAUVIN, Danièle et SIGANOS, André et WALTER, Philippe, éditions Imago, Paris, 2005.

ROCQUET, Jean-Paul, Lecture et implicite, Novembre 2002.

SALLENAVE, Danièle, Le Don des morts sur la littérature. Gallimard, Paris, 1991.

TAUVERON, Catherine, Lire la littérature à l'école : Pourquoi et comment conduire cet apprentissage spécifique? De la GS au CM, Hatier, France, 2002. 
TAUVERON, Catherine, Le Personnage : une clef pour la didactique du récit à l'école élémentaire,

Delachaux et Niestlé, Neuchâtel, Paris, 1995.

TODOROV, Tzvetan, La Littérature en péril, Flammarion, Paris janvier 2007.

TOURSEL, Nadine et VASSEVIERE, Jacques, Littérature : textes théoriques et critiques, Armand Colin (2 2 ème

édition), Paris, 2010[2008].

\section{NOTES}

1. Selon l'Encyclopédie Universalis en ligne "en textile, type de tissu africain à décors peints par teinture». C'est aussi une technique d'impression textile originaire du Mali et pratiquée sur des cotonnades entièrement artisanales. Elle consiste en une teinture des tissus en aplats qui ménage des réserves de tissu; ces « vides » constituent paradoxalement la couleur.

2. L'expression est de François Mauriac, «Le romancier et ses personnages», 1933. Dans l'approche formaliste, le personnage n'est qu'un signe textuel et le produit du contexte intellectuel des années 1960.

3. Le premier aspect, précédemment soulevé, est relatif au métissage linguistique et à l'hybridité textuelle qui caractérisent les textes contemporains.

4. Disponible sur : https://arlap.hypotheses.org/1738

5. Entendue comme une organisation/attribution des valeurs. C'est justement là que le mythe, étant une forme narrative, aide à mettre en évidence la question des représentations socioculturelles lors d'une lecture littéraire.

6. Un questionnaire a été distribué aux étudiants avant l'engagement dans les séances du débat. Les questions tournaient autour de certains renseignements personnels (sexe, âge, fonction, résidence, vocation, loisirs, lectures faites...).

7. "Née dans les années soixante-dix, la mythocritique s'inscrit dans le champ de la "nouvelle critique». Son promoteur, Gilbert Durand, forge le terme sur le modèle de la psychocritique de Charles Mauron. Mais à l'inverse de la psychocritique, où une approche particulière est appliquée à un objet, il s'agit apparemment dans la mythocritique d'appliquer un objet à un autre objet, de lire le texte sous l'angle du mythe, un récit à travers un récit. » (RIALLAND, Ivanne, "La mythocritique en questions ", in Questions de Mythocritique. Dictionnaire, sous la direction de CHAUVIN, Danièle / SIGANOS, André / WALTER, Philippe, éditions Imago, Paris, 2005).

8. Le concept d'« isotopie " a été proposé par A.J Greimas : il y a isotopie, lorsque les signes textuels renvoient à un même lieu. Il s'agit d'« un ensemble redondant de catégories sémantiques qui rend possible la lecture uniforme du récit, telle qu'elle résulte des lectures partielles des énoncés et de la résolution de leurs ambiguités qui est guidée par la recherche de la lecture unique. » (A. J. Greimas, Du sens -Essais sémiotiques, éditions du Seuil, 1970. Cité par A. HÉNAULT, 1993, 91).

\section{RÉSUMÉS}

Par son inscription dans une dimension ontologique et anthropologique, par sa faculté de conduire son lecteur vers un processus d'identification, le mythe se révèle une clé pour la lecture, la compréhension et l'interprétation des œuvres littéraires. Les mythes sont d'universels 
vecteurs de symbolisation (FOURTANIER, 2012 : 23-24). En ce sens, quels effets pourrait introduire une dé(re)contextualisation du mythe quant à sa réception? Quel rôle peuvent jouer les stéréotypes culturels dans la réception et l'interprétation des mythes? Un personnage jugé immoral, fantastique ou absurde, pourrait-il nous influencer? Pourquoi et comment nos attitudes changent envers les personnages fictifs?

La lecture des mythes est un espace où peuvent s'articuler tous les compromis. Capitale qu'elle soit exercée au sein des classes de FLE : sa prise en charge doit tenir en compte la dyade (posture $\mathrm{du}$ lecteur et univers symbolico-culturel du texte).

By its inscription in an ontological and anthropological dimension, by its ability to lead its reader towards an identification process, the myth is a key to the reading, the comprehension and the interpretation of literary works. Myths are universal vectors of symbolization (FOURTANIER, 2012 : 23-24). In this sense, what effects could a (re) contextualization of the myth introduce to its reception? What role can cultural stereotypes play in the reception and interpretation of myths? Could a character considered immoral, fantastic or absurd, influence us ? Why and how do our attitudes change towards fictional characters?

The reading of myths is a space where all compromises can be articulated. Capital that it is exercised within FFL classes : its support must take into account the dyad (posture of the reader and symbolic-cultural universe of the text).

\section{INDEX}

Keywords : reception, myth, comparative reading, stereotype, profile

الاستقبال, الأسطورة, القراءة المقارنة, الصورة النمطية, الملففهرس الكلمات المفتاحية:

Mots-clés : réception, mythe, lecture comparative, stéréotype, profil

\section{AUTEUR}

WASSILA SOLTANI

Université de Biskra 